\title{
Fractals with optimal information dimension
}

\author{
Subhash Kak \\ Oklahoma State University, Stillwater \\ Email: subhash.kak@okstate.edu
}

\begin{abstract}
We present novel fractals with dimension that is close to the optimal value of $e$. The examples are for up to 10-way branching at each iteration and they include symmetric and asymmetric ones, as well as those where in addition to holes a few layers of other regions are peeled. Only representative fractals are described and the method can be used to find many other designs. Since natural systems are likely to be optimal, the examples have potential applications in both natural or engineered systems.
\end{abstract}

\section{Introduction}

Many complex phenomena are associated with fractals -- objects with salient features of self-similarity and scaling [1] -- that have noninteger dimensionality. The selfsimilarity may be approximate, exact, or be defined statistically; it can also be associated with time series [2]. Recent papers show how the optimal dimensionality of $e$ characterizes physical space [3-5], which opens up new applications to engineered systems. The application of fractals to physical systems, astronomy, complex and social networks, medicine and biology has been investigated for many years [6-9]. New results on data representation $[10,11]$ and the fact $e$ represents the intrinsic dimensionality of data [12] opens up applications to situations with large data.

The optimum dimensionality of $e$ may be viewed in terms of the structure of space or complementarily as creating potentials that lead to specific self-similar and scaleinvariant structures [3]. Physical space is isotropic, so if it is $e$ dimensional, the corresponding two-dimensional and one-dimensional sets will have measures of $2 e / 3$ and $e / 3$.

In this paper, we look at new recursive sets that nearly equal optimal values and are likely to be encountered in engineered and natural systems. We present recursive constructions that show how optimal fractals may be obtained (the examples presented here are only representative) and these constructions may be used to find further examples. The designs presented include hybrid ones where each iteration includes holes and peeled layers.

Since the fractals presented here are novel, they are also of pedagogic interest. 


\section{Background}

The box-counting dimension of a fractal is defined as:

$$
\operatorname{dim}_{\text {box }}(S)=d=\lim _{\epsilon \rightarrow \infty} \frac{\ln N(\epsilon)}{\ln \left(\frac{1}{\varepsilon}\right)}
$$

It is assumed that the set is generated by a recursive algorithm where each "box" is replaced by $N(\epsilon)$ scaled copies at each iterative step, $\frac{1}{\varepsilon}=n$. The dimension $d$ may be written as:

$$
d=\frac{\ln N(\epsilon)}{\ln n}
$$

For optimum information representation $[3,12], d=e$.

When $d=e / 3, N(\epsilon)=n^{e / 3}$. When there is a two-way iteration, that is $n=2$,

$$
d_{1}=\frac{\ln 1.874}{\ln 2} \approx 0.906
$$

This is the optimal dimension on the line.

For a corresponding two-dimensional surface, $d=2 e / 3, N(\epsilon)=n^{2 e / 3}$. Therefore (for $n=4)$,

$$
d_{2}=\frac{\ln 12.322}{\ln 4} \approx 1.812
$$

This is the optimal dimension on the plane.

For $d=e, N(\epsilon)=n^{e}$, that is the three-dimensional case

$$
d_{3}=e \approx \frac{\ln 6.581}{\ln 2} \approx 2.718
$$

Note that the fractals may be symmetric or asymmetric with respect to the principal axes of the cover space. The fractals encountered in Nature will be isometric and will not conform to such constraints of symmetry.

\section{1- and 2-D cases}


Table 1 provides the relevant numbers for the 1-D and 2-D cases for $n \leq 10$, where $n^{e / 3}$ and $n^{2 e / 3}$ are compared with $n$ and $n^{2}$, to see which integer values can be extracted from the perspective of generating new fractals.

Table 1. First iteration cubes $n^{e / 3}$ and $n^{2 e / 3}$ for different $n$

\begin{tabular}{|c|c|c|c|}
\hline$n$ & $n^{e / 3}$ & $n^{2 e / 3}$ & $n^{2}$ \\
\hline 2 & 1.874 & 3.511 & 4 \\
\hline 3 & 2.706 & 7.322 & 9 \\
\hline 4 & 3.512 & 12.322 & 16 \\
\hline 5 & 4.299 & 18.478 & 25 \\
\hline 6 & 5.071 & 25.713 & 36 \\
\hline 7 & 5.831 & 33.999 & 49 \\
\hline 8 & 6.581 & 43.310 & 64 \\
\hline 9 & 7.322 & 53.612 & 81 \\
\hline 10 & 8.055 & 64.890 & 100 \\
\hline
\end{tabular}

The most convenient choices of $n$ for the line are 6 and 10, and for the plane are 7 and 10 , as the difference between $n$ and $n^{e / 3}$ for the line, and the difference between $n^{2}$ and $n^{2 e / 3}$ for these values is small.

The fractal for one-dimension with 6-way subdivision will be:

\section{Asymmetric line fractal}

1. Divide unit length into 6 parts and drop, say, the third part. (We can choose to drop any of the six, so long it is done at each iterative step.

2. Take each subpart, and divide further into 6 sub-sub-parts, dropping one, and continue.

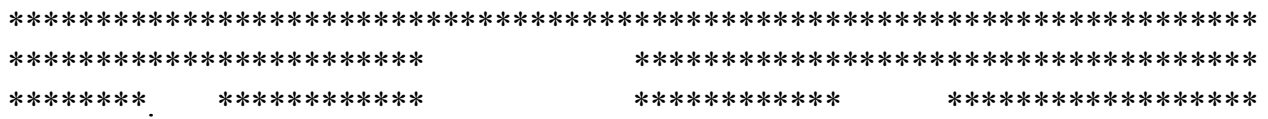

Figure 1. One dimensional fractal with 6-way subdivision

Its dimensionality is:

$$
d=\frac{\ln 5}{\ln 6} \approx 0.898
$$

This value is a bit less than the optimal value of 0.906 for the line. 
The more efficient line fractal is for $n=10$, in which specified 2 sub-parts (out of a total of 10$)$ are removed in each iteration, giving us the better value:

$$
d=\frac{\ln 8}{\ln 10} \approx 0.903
$$

Nearly symmetric 2-D fractal

For the fractal in two-dimension with 7-way subdivision, we choose the following procedure:

1. Divide unit square into $7 \times 7=49$ parts and drop 15 sub-squares (since we need to retain 34 ). This can be done in a variety of ways, and we propose to do it as in Figure 2.

2. Take each subpart, and divide further into 49 sub-sub-parts, and continue. Its

$$
d=\frac{\ln 34}{\ln 7}=1.812,
$$

which is nearly equal to the optimal value.

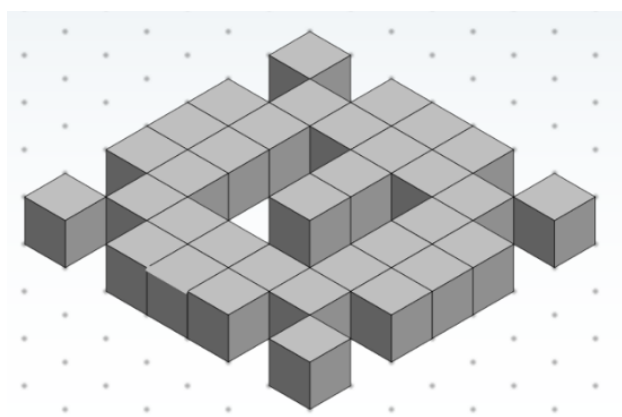

Figure 2. The optimal fractal iteration in 2-dimensions

Note it is symmetric in the two principal directions except for one square next to the one in the middle of the arrangement.

For $n=10$, the simple fractal that removes 35 squares out of 100 (which can be done by taking them out in a rectangular area of $5 \times 7$ that is offset in its symmetry by one square, gives

$$
d=\frac{\ln 65}{\ln 10} \approx 1.813
$$

that is even closer to the optimal value. 


\section{The three-dimensional case}

For the three-dimensional case, one must consider the difference between $n^{3}$ and $n^{e}$. Figure 3 shows that the ratio $\frac{n^{e}}{n^{3}}$ representing the density falls below 0.5 for $n$ just over 11.

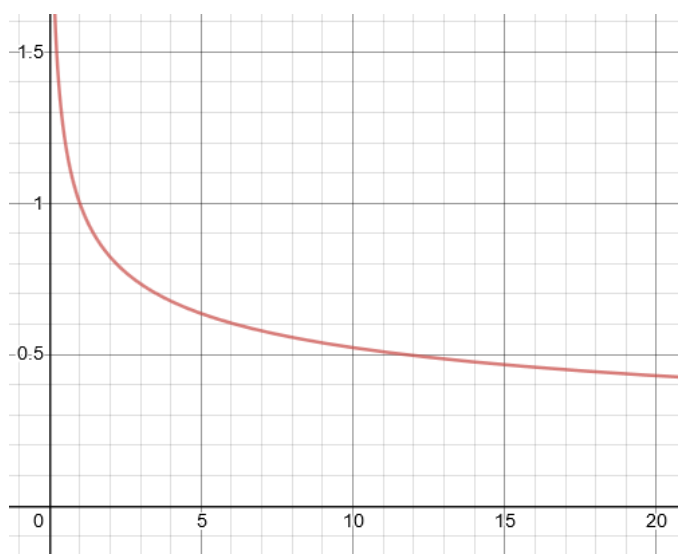

Figure 3. The relationship of $\frac{n^{e}}{n^{3}}$ with respect to $n$

We must pick a value of $n$ for which the difference between $n^{3}$ and $n^{e}$ is very close to an integer (Table 2).

Table 2. First iteration cubes $n^{e}$ for different $n$

\begin{tabular}{|c|c|c|c|}
\hline$n$ & $n^{e}$ & $n^{3}$ & $\left\lfloor n^{3}-n^{e}\right\rfloor$ \\
\hline 2 & 6.581 & 8 & 1 \\
\hline 3 & 19.813 & 27 & 7 \\
\hline 4 & 43.308 & 64 & 21 \\
\hline 5 & 79.432 & 125 & 46 \\
\hline 6 & 130.387 & 216 & 86 \\
\hline 7 & 198.251 & 343 & 145 \\
\hline 8 & 285.005 & 512 & 227 \\
\hline 9 & 392.555 & 729 & 336 \\
\hline 10 & 522.735 & 1000 & 477 \\
\hline
\end{tabular}

\section{A constructive procedure}

Remove area of $k_{1} \times k_{2}$ sub-cubes from a side together with the ones that lie beneath all the way through, and replicate this along the other two axes.

Theorem. The total number of sub-cubes removed from the large cube of side $n$ when $k_{1} \times k_{2}$ sub-cube hole is created in each side is: 


$$
3 k_{1} k_{2} n-k_{1}^{2} k_{2}-k_{1} k_{2}^{2}
$$

Proof. Since the area of the sub-cubes from each side is $k_{1} k_{2}$ and the depth is $n$, the volume removed looking from each side is $k_{1} k_{2} n$ for a total for the three axes equaling $3 k_{1} k_{2} n$, from which we should subtract the numbers that are counted more than once. As seen from the example of Figure 4 , where $k_{1}=1, k_{2}=2$, the overlap in the empty space is either along $k_{1}$ or $k_{2}$; therefore, the overcount is $k_{1}^{2} k_{2}+k_{1} k_{2}^{2}$ which is to be subtracted.

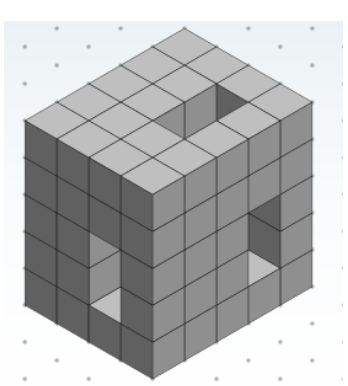

Figure 4. Asymmetric sub-cube removal

For the symmetric case where the middle $k^{2}$ sub-cubes are removed from each side together with those that lie under them, the number removed (Figure 5) is clearly:

$$
k^{2} n+2 k^{2}(n-k)=3 k^{2} n-2 k^{3}
$$

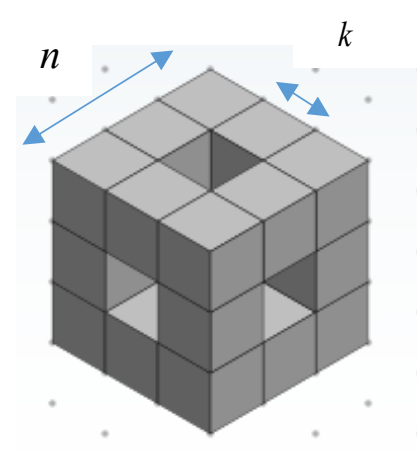

Figure 5. The fractal with middle $k^{2}$ sub-cubes removed

This corresponds to the case where $k_{1}=k_{2}=k$ in equation (10).

$n=2 ;$ Alternating steps

One can choose the construction of Figure 6 , alternating between 6 and 7 cubes out of 8 in the iterative process. 


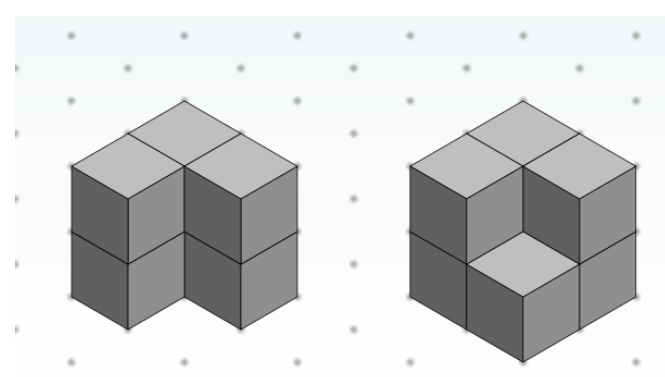

Figure 6. Construction for $n=2$.

We can take the average number of sub-cubes to be 6.5. The dimension, $d$, will thus be:

$$
d=\frac{\ln 6.5}{\ln 2} \approx 2.700
$$

$n=3$; Menger sponge

This case $n=3$ corresponds to the familiar Menger sponge $[1,2,13,14]$, where in each iteration the middle sub-cube of each side and the innermost sub-cube (for a total of 7) are left out, thus keeping 20.

$$
d=\frac{\ln 20}{\ln 3} \approx 2.727
$$

This is symmetrical with respect to the six directions associated with the cube.

$n=4$; Corkscrew

From Table 2, we know that we need to retain 43.30 sub-cubes out of 64 .

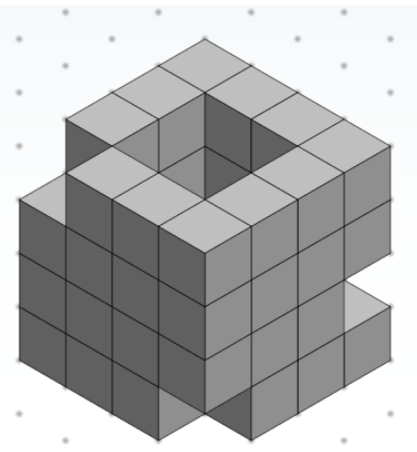

Figure 7. Construction for $n=4$.

Figure 7 shows an asymmetric way of doing it, where 16 sub-cubes in the middle, and 4 corners cubes of the four layers have been removed. This leaves us with 44 sub-cubes, with a corresponding dimension of 


$$
d=\frac{\ln 44}{\ln 4} \approx 2.730
$$

If we remove 20 sub-cubes in one iteration and 21 sub-cubes in the following iteration in an alternating manner, the average number of sub-cubes removed can be taken to be 20.5 , so that the dimension will now be:

$$
d=\frac{\ln 43.5}{\ln 4} \approx 2.721
$$

For a more regular, Menger sponge-like solution, we can pick a window of $2 \times 1$ and remove $3 k_{1} k_{2} n-k_{1}^{2} k_{2}-k_{1} k_{2}^{2}=18$ sub-cubes (Figure 8), with a dimension of $d=$ $\frac{\ln 46}{\ln 4} \approx 2.762$.

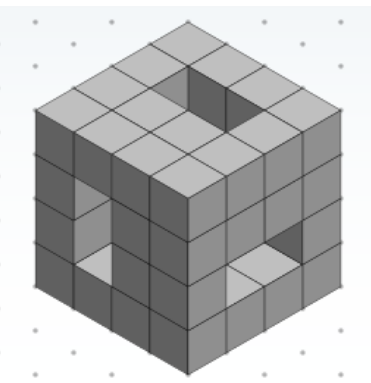

Figure 8. Regular symmetric construction for $n=4$.

$n=5$. Tube.

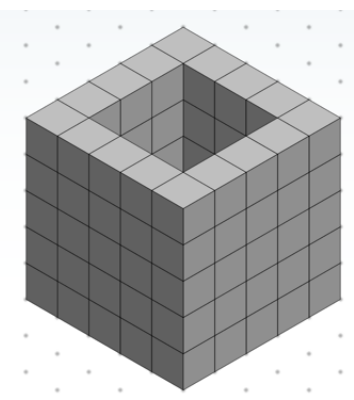

Figure 9. Construction for $n=5$.

Here we have chosen a completely asymmetric fractal where the inner cubes have all been removed along one axis.

$$
d=\frac{\ln 80}{\ln 5} \approx 2.723
$$


A more regular structure with 4 out of 25 removed on each face and all the way through for a total of $3 k^{2} n-2 k^{3}=60-16=44$ sub-cubes (Figure 10), for $d=\frac{\ln 81}{\ln 5} \approx 2.730$

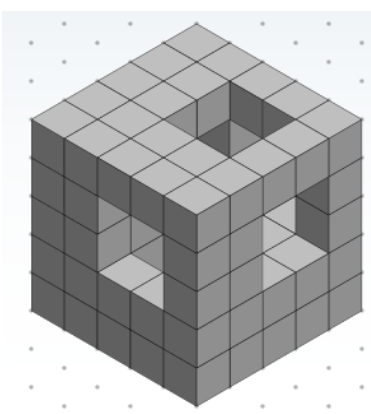

Figure 10. Construction for $n=5$.

$n=8$. Hole plus Peel

The closest match in Table 2 is for $n=8$. The algorithm to generate it must remove 227 sub-cubes, which equals 38 cubes each side and one less. One can also have an algorithm that alternates between two constructions.

A simple way to remove 228 sub-cubes is to have holes go through each side of area $3 \times 4$ and just peel 4 outer-layer sub-cubes in each side (shown in Figure 11 by white and blue squares, respectively). The holes of sides $3 \times 4$ correspond to 204 sub-cubes, and the 4 outer-layer peeled sub-cubes for 6 sides total 24, for an aggregate of $228 \mathrm{sub}$ cubes. With $512-228=284$ sub-cubes remaining,

$$
d=\frac{\ln 284}{\ln 8} \approx 2.717
$$

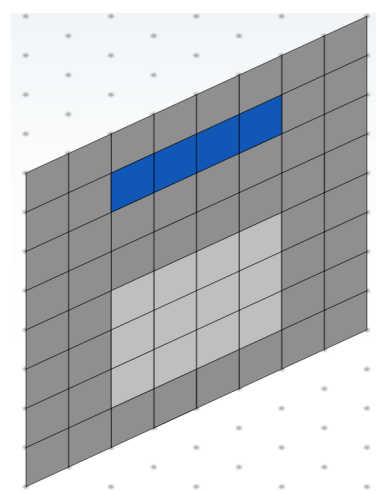

Figure 11. Construction for $n=8$ for each side (blue: outer layer sub-cubes peeled; white: sub-cubes through to the other side).

n=9. Peel only 
The sub-cubes to be removed is 336 (Table 2 ). This can be easily done by peeling off 56 sub-cubes from each face $(336 / 6=56)$, which can be done by a square of $7 \times 8$ located as convenient. The dimension will be

$$
d=\frac{\ln 393}{\ln 9} \approx 2.719
$$

Another solution somewhat like Figure 11 is a hole of $4 \times 4$ on each side (reduces subcubes by 304), together with peeling of 5 sub-cubes from outer surface of each side for a total of 30 , with aggregate of 334 . This gives us:

$$
d=\frac{\ln 395}{\ln 9} \approx 2.721
$$

The figures of equation 18 and 19 are closer to the optimum as compared to the value of 2.727 for the Menger sponge.

$n=9$. Hollow

If the inner structure of 7 sub-cubes, which is enclosed by a layer of sub-cubes on all sides giving us the edge size of 9 , is removed, then the total number removed will be $7^{3}=343$, leaving us with $729-343=386$. The dimension of this fractal where at each step the inner $7 / 9$ th of the cube is removed will be:

$$
d=\frac{\ln 386}{\ln 9} \approx 2.711
$$

$n=10$. Hole plus peel

One needs to remove 477 sub-cubes for optimality (Table 2). For a solution like Figure 11 , each side has a hole of $4 \times 5$ (= 420 total) and a one-layer peel from each side of $3 \times 3$ (= 54 total) for aggregate of 474 . Therefore, this fractal has

$$
d=\frac{\ln 526}{\ln 10} \approx 2.721
$$

The approach outlined here can be used to create many other novel fractals. Rectangular holes can be distributed in smaller sized holes across the side providing flexibility in the design.

\section{Conclusions}

The paper presented new fractals that are optimum or near-optimum with respect to information efficiency. Since Nature is optimal, these fractals have potential applications in noninteger dimensional natural or engineered systems. The novel 
structures found include those with asymmetric holes, and combination of holes and peels.

\section{REFERENCES}

1. Mandelbrot, B. B., The Fractal Geometry of Nature. (W. H. Freeman, 1983).

2. Falconer, K.J., Fractal Geometry: Mathematical Foundations and Applications. (Wiley, 2003).

3. Kak, S. Information theory and dimensionality of space. Sci Rep 10, 20733 (2020). https://doi.org/10.1038/s41598-020-77855-9

4. Kak, S. Noninteger dimensional spaces and the inverse square law. (2020); TechRxiv: https://www.techrxiv.org/articles/preprint/Noninteger Dimensional Spaces and the In verse Square Law/13079720

5. Kak, S. Asymptotic freedom in noninteger spaces. (2020). TechRxiv: https://www.techrxiv.org/articles/preprint/Asymptotic Freedom in Noninteger Dimensi onal Spaces/13270556

6. Garrido, E., Jensen, A.S., Confinement of two-body systems and calculations in d dimensions. Phys. Rev. Research 1, 023009 (2019)

7. Sreenivasan, K.R. Fractals and multifractals in fluid turbulence. Annual Rev. Fluid Mech. 23, 539-600 (1991)

8. Kak, S. Power series models of self-similarity in social networks. Information Sciences 376 , 31-38 (2017)

9. Losa, G.A., Nonnenmacher, T. F., eds. Fractals in Biology and Medicine. Springer (2005)

10. Kak, S. Information, representation, and structure. International Conference on Recent Trends in Mathematics and Its Applications to Graphs, Networks and Petri Nets, New Delhi, India (2020).

11. Kak, S. The base-e representation of numbers and the power law. Circuits Syst. Signal Process. (2020) https://doi.org/10.1007/s00034-020-01480-0

12. Kak, S. The intrinsic dimensionality of data. Circuits Syst. Signal Process. (2020); https://doi.org/10.1007/s00034-020-01583-8

13. Menger, K., Reminiscences of the Vienna Circle and the Mathematical Colloquium. (Springer Science \& Business Media, 2013).

14. Bunde, A., Havlin, S., Fractals in Science. (Springer Science \& Business Media, 2013). 